\title{
A Multi-antenna Iterative Frequency-domain Detection for Power-efficient NOMA Schemes
}

\author{
João Guerreiro ${ }^{1,2}$, Filipe Casal Ribeiro ${ }^{1}$, Rui Dinis ${ }^{1,3}$, Paulo Montezuma ${ }^{1,3}$, \\ Dushantha Jayakody ${ }^{4}$, and Mário Marques da Silva ${ }^{1,2}$ \\ ${ }^{1}$ IT — Instituto de Telecomunicações, Portugal \\ ${ }^{2}$ Universidade Autónoma de Lisboa, Portugal \\ ${ }^{3}$ FCT - Universidade Nova de Lisboa, Portugal \\ ${ }^{4}$ National Research Tomsk Polytechnic University, Russia
}

\begin{abstract}
The transmitted signals in non-orthogonal multiple access (NOMA) schemes can have substantial envelope fluctuations, not to mention the inherent high interference levels. This means that a high price in terms of power efficiency can be payed to achieve the substantial capacity gains of NOMA systems. In this paper we consider the use single-carrier signals combined with NOMA schemes in the downlink transmission. We present a multi-antenna transmission technique that is compatible with the use of high-efficiency power amplifiers and does not require any pre-processing and allows the use of power-efficient amplifiers. Moreover, an efficient detection is achieved using iterative receivers with joint multi-user detection and equalization. Our performance results show that the proposed technique allows high power efficiency, with bit-error-rate (BER) performance close to the matched filter bound (MFB).
\end{abstract}

\section{INTRODUCTION}

Non-orthogonal multiple access (NOMA) schemes [1,2] allow substantial capacity gains, since multiple users can share the same physical channel. For this reason, they are being considered for future $5 \mathrm{G}$ systems [3-7].

The most common NOMA approach is to transmit simultaneously signals with different power levels and to take advantage of this power difference for their separation, since the low power signal is designed to produce negligible interference in the high power signal and the high power signal can be estimated accurately and removed when detecting the low power signal [8]. This is particularly interesting for the downlink, with the base station (BS) transmitting simultaneously to different users that are placed at different distances and, consequently, have different transmit power requirements due to different propagation losses [9].

However, NOMA schemes have implementation difficulties, not only associated to the users' separation when the power difference between users in not high enough, but also because the signal to be transmitted can have high envelope fluctuations. In fact, we need to employ very linear amplifiers, with reduced amplification efficiency, even when single-carrier (SC) signals and constant-envelope constellations (e.g., quadrature phase shift keying (QPSK) constellations) are employed.

In this paper we consider the use of single-carrier signals combined with NOMA schemes for the downlink transmission. We present a multi-antenna transmission technique that does not require pre-processing and allows the use of power-efficient amplifiers. The basic idea is to have a different amplifier and antenna for each user. This means that we can employ signals with reduced envelope fluctuations for each user (e.g., QPSK signals with reduced envelope fluctuations such as the ones of [10], or even constant envelope signals based on offset modulations [11]). Since the signals are combined in the channel, there are no combining losses. We also employ an efficient iterative frequency-domain receiver for joint equalization and cancellation of signals associated to stronger users.

This work is organized as follows: Section 2 describes the considered downlink NOMA system and characterizes the transmitted signals. Section 3 is focused on the receiver design regarding the different NOMA users. In Section 4 a set of performance results is presented and Section 5 concludes the paper.

In this paper we adopt the following notations: bold upper case letters denote matrices or vectors; $\mathbf{I}_{N}$ denote the $N \times N$ identity matrix; $\mathbf{x}^{*}, \mathbf{x}^{T}$ and $\mathbf{x}^{H}$ denote complex conjugate, transpose and hermitian (complex conjugate transpose) of $\mathbf{x}$, respectively. In general, lower case letters denote 
time-domain variables and upper case letters denote frequency-domain variables; $\tilde{x}, \hat{x}$ and $\bar{x}$ denote the equalized signal, the "hard decisions" and the "soft decisions" estimates of $x$, respectively. The expectation of $x$ is denoted by $\mathbb{E}[x]$.

\section{SYSTEM CHARACTERIZATION}

The system is characterized by the implementation of NOMA schemes combined with single-carrier with frequency-domain equalization (SC-FDE) modulation signals. We consider the downlink transmission of such schemes, where a multiple antenna BS transmits simultaneously signals with different power levels to $P$ single-antenna mobile terminals at distinct distances.

Without loss of generalization, we consider $P=2$ MTs and a BS with $T=2$ transmit antennas as shown in Fig. 1. Note that as the MTs are at different distances, the BS adapts the power level for each MT. Therefore, the BS should transmit with low power for the closest MT and with high power for the furthest MT. ${ }^{1}$ In traditional NOMA schemes, a single transmission antenna is adopted to transmit the $P$ signals. Thus, even with constant envelope constellations, the superposition of these signals leads to a signal with large envelope fluctuations, which brings amplification difficulties. In fact, in order to avoid nonlinear distortion effects, the power amplifier should: (i) have a large linear dynamic range to accommodate the high power signal and (ii) operate with a large input back-off. Under these two conditions, the amplification efficiency can be very low, which is a considerable disadvantage of NOMA. In this work, we employ a different approach where each one of the $P$ signals is amplified and transmitted by a separate antenna. This means that we can adopt constellations with reduced envelope fluctuations, combined with power-efficient, nonlinear amplifiers (NLAs) that, although work with different output powers, can operate in the saturation regime to achieve a very high amplification efficiency [12]. In addition, since the signal is combined at the channel level, we de not have combination losses.

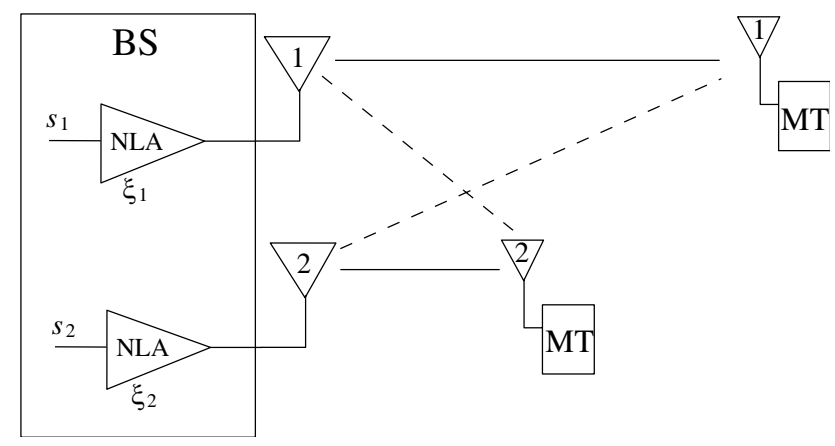

Figure 1: Adopted cellular scenario.

We consider SC-FDE modulations [13] where each transmitted block has $N$ data symbols. The signal transmitted for the $p$ th user is defined as $\mathbf{s}_{p}=\left[\begin{array}{llll}s_{0, p} & s_{1, p} & \ldots & s_{N-1, p}\end{array}\right]^{T}$, where $s_{n, p}$ is a QPSK symbol. The channels between the MTs and each BS transmit antenna are frequency-selective with $I$ uncorrelated Rayleigh multipath components. We define the channel between the $p$ th MT and the $t$ th transmit antenna for the $k$ th subcarrier as $H_{k}^{(t, p)}\left(\mathbb{E}\left[\left|H_{k}^{(t, p)}\right|^{2}\right]=1\right)$, and we consider two scenarios: one where the antennas transmit antennas have a little separation the channels between the BS and the $P$ MTs are equal, i.e., $H_{k}^{(1, p)}=H_{k}^{(2, p)}$, and another where the antennas have a higher separation and the channels are different and uncorrelated.

Thanks to a cyclic prefix larger than the channel impulsive response, we can express the received signal for the $p$ th MT and subcarrier as

$$
Y_{k, p}=\sum_{t=1}^{T} \xi_{p} S_{k, t} H_{k}^{(t, p)}+N_{k},
$$

with $S_{k, p}$ and $N_{k}$ denoting the frequency-domain symbol transmitted to the $p$ th $\mathrm{MT}$ and the additive white Gaussian noise (AWGN) component associated to the $k$ th frequency, with variances $\mathbb{E}\left[\left|S_{k, p}\right|^{2}\right]=2 \sigma_{S}^{2}$ and $\mathbb{E}\left[\left|N_{k}\right|^{2}\right]=2 \sigma_{N}^{2}$, respectively. The inherent power control of NOMA systems

\footnotetext{
${ }^{1}$ In the following, we refer to the "high-power signal" as the signal transmitted fo the furthest user and the "low-power signal" as the signal transmitted for the closest user.
} 
involves the adoption of different amplifications gains at the NLAs of the BS. The amplification gain of the $p$ th NLA is denoted by $\xi_{p}$. It should also be mentioned that index $p$ is related to the distance between the MT and the BS: the higher the distance the lower the value of $p$, which means that in our NOMA scheme we have $\xi_{1}>\xi_{2}$. In that context, we define the power separation ratio (PSR) between the two users as

$$
\beta=20 \log _{10}\left(\frac{\xi_{1}}{\xi_{2}}\right) .
$$

Clearly, the PSR should be high enough to allow for the user separation.

\section{RECEIVER DESIGN}

In NOMA systems, the power domain is used for performing the users separation. In this work, we adopt an FDE-based reception for multi-user detection and interference cancellation and we make use of two facts: (i) the low-power signal produces a small interference in the high power signal and (ii) the high power signal can be easily detected and subtracted from the low power signal. Therefore, although the data detection of both users is based on traditional iterative block decision feedback equalization (IB-DFE) $[13,14]$, they have some differences that will be explained in the following subsections.

\subsection{Furthest User}

Let us start by describing the detection of the high power signal, which is addressed to the furthest user. In this case, as the low-power signal is designed to produce a negligible interference on the high power signal, the received signal can be approximated by

$$
Y_{k, 1}=\sum_{t=1}^{T} S_{k, 1} H_{k}^{(t, 1)}+N_{k} \approx S_{k, 1} H_{k}^{(1,1)}+N_{k} .
$$

This approximation is justified by the use of an adequate PSR. In fact, it is very tight as long as the PSR is high enough to neglect the interference from the low-power signal. Therefore, the detection of the furthest user can be seen as a single-input, single-output (SISO) IB-DFE scenario, where an IB-DFE detection with $L$ iterations is performed. Under these conditions, the equalized signal associated to the $k$ th subcarrier at the $l$ th iteration is given by

$$
\tilde{S}_{k, 1}^{(l)}=F_{k}^{(l)} Y_{k, 1}-B_{k, 1}^{(l-1)} \bar{S}_{k, 1}^{(l-1)}
$$

where $\bar{S}_{k, p}^{(l)}$ is the DFT of the block of time-domain average values conditioned to the detector output $\bar{s}_{n, p}$, also denoted as "soft-decisions". For normalized QPSK constellations, i.e., $s_{n, p}= \pm 1 \pm j$, these average values are defined as

$$
\bar{s}_{n, p}=\tanh \left(\frac{L_{n, p}^{R e}}{2}\right)+j \tanh \left(\frac{L_{n, p}^{I m}}{2}\right),
$$

where $L_{n, p}^{R e}=\frac{2}{\sigma_{n, p}^{2}} \operatorname{Re}\left(\tilde{s}_{n, p}\right), L_{n, p}^{I m}=\frac{2}{\sigma_{n, p}^{2}} \operatorname{Im}\left(\tilde{s}_{n, p}\right)$, and

$$
\begin{aligned}
\sigma_{n, p}^{2} & =\frac{1}{2 N} \sum_{n^{\prime}=0}^{N-1}\left|\tilde{s}_{n^{\prime}, p}-s_{n^{\prime}, p}\right|^{2} \\
& \simeq \frac{1}{2 N} \sum_{n^{\prime}=0}^{N-1}\left|\tilde{s}_{n^{\prime}, p}-\hat{s}_{n^{\prime}, p}\right|^{2} .
\end{aligned}
$$

The feedforward and the feedback equalization coefficients of the IB-DFE are defined respectively as

$$
F_{k}^{(l)}=\frac{H_{k}^{*(1,1)}}{\left(1-\rho^{(l-1)^{2}}\right) \sum_{t=1}^{2}\left|H_{k}^{(t, 1)}\right|^{2}+\frac{1}{\mathrm{SNR}}},
$$


and

$$
B_{k}^{(l)}=\rho^{(l-1)}\left(F_{k}^{(l)} H_{k}^{(1,1)}-1\right),
$$

where the parameter $\rho^{(l)}$ measures the reliability of the data estimates of the $l$ th iteration (it is assumed that $\rho^{(0)}=0$, which means that when $l=1$ we have a conventional, linear FDE), and is defined as

$$
\rho^{(l)}=\frac{1}{2 N} \sum_{n=0}^{N-1}\left(\left|\operatorname{Re}\left(\bar{s}_{n, 1}^{(l)}\right)\right|+\left|\operatorname{Im}\left(\bar{s}_{n, 1}^{(l)}\right)\right|\right) .
$$

\subsection{Closest User}

As mentioned before, the low power user performs an IB-DFE technique with $L$ iterations to detect its own signal. In addition, it must perform and IB-DFE technique to detect the signal interfering signal addressed to the furthest user. In fact, this means that this detection scenario can be seen as a multiple-input, single-output (MISO) IB-DFE system, where a successive interference cancellation (SIC) is employed for joint detection and equalization. Therefore, at the $l$ th iteration, the closest user estimates the high power signal as ${ }^{2}$

$$
\tilde{S}_{k, 1}^{(l)}=F_{k, 1} Y_{k, 2}-B_{k, 1}^{(l)} \bar{S}_{k, 1}^{(l-1)}-\rho_{2}^{(l)} F_{k, 1} H_{k, 2} \bar{S}_{k, 2}^{(l)},
$$

where

$$
F_{k, 1}^{(l)}=\frac{H_{k}^{*(1,2)}}{\left(1-\rho_{1}^{(l-1)^{2}}\right) \sum_{t=1}^{2}\left|H_{k}^{(t, 2)}\right|^{2}+\frac{1}{\mathrm{SNR}}}
$$

and

$$
B_{k, 1}^{(l)}=\rho_{2}^{(l-1)}\left(F_{k, 1}^{(l)} H_{k}^{(1,2)}-1\right) .
$$

On the other hand, at the $l$ th iteration, the low-power signal (which is the desired data signal) is detected as

$$
\tilde{S}_{k, 2}^{(l)}=F_{k, 2} Y_{k, 2}-B_{k, 2}^{(l)} \bar{S}_{k, 2}^{(l-1)}-\rho_{1}^{(l)} F_{k, 2} H_{k, 1} \bar{S}_{k, 1}^{(l)},
$$

where

$$
F_{k, 2}^{(l)}=\frac{H_{k}^{*(2,2)}}{\left(1-\rho_{2}^{(l-1)^{2}}\right) \sum_{t=1}^{2}\left|H_{k}^{(t, 2)}\right|^{2}+\frac{1}{\mathrm{SNR}}},
$$

and

$$
B_{k, 2}^{(l)}=\rho_{2}^{(l-1)}\left(F_{k, 1}^{(l)} H_{k}^{(2,2)}-1\right) .
$$

The reliability of the data associated to the $p$ th data stream (desired data or interference from the furthest user) is computed as in (9). For both users, the estimated signal at the $l$ th iteration is

$$
\hat{s}_{n, p}^{(l)}=\operatorname{sign}\left(\operatorname{Re}\left(\tilde{s}_{n, p}^{(l)}\right)\right)+j \operatorname{sign}\left(\operatorname{Im}\left(\tilde{s}_{n, p}^{(l)}\right)\right),
$$

where the $\operatorname{sign}(\cdot)$ function is used to obtain the hard-decisions.

\section{PERFORMANCE RESULTS}

In this section we present a set of results regarding the performance of the proposed NOMA system with iterative FDE receivers. Otherwise stated, we consider SC blocks with $N=256$ QPSK data symbols selected under Gray mapping as well as frequency-selective channels with $I=64$ uncorrelated multipath components with Rayleigh fading.

Figure 2 shows the bit error rate (BER) associated with the furthest user considering both equal and uncorrelated channels between the BS antennas and that user, IB-DFE receiver with $L=4$ iterations and a PSR of $\beta=14 \mathrm{~dB}$. From the figure, it can be noted that neglecting the low-power

\footnotetext{
${ }^{2}$ Note that this estimation is different from the one performed by the furthest user since the low-power signal, that represents inter-user interference (IUI), is subtracted from the estimation of the high-power signal, i.e., there is not only ISI cancellation but also IUI cancellation.
} 
signal has a reduced impact on the performance of the IB-DFE detection of the high-power signal, since the after $L=4$ iterations the performance is close to the MFB, as happens in conventional single-user IB-DFE systems. This means that a PSR of $\beta=14 \mathrm{~dB}$ is enough to separate the two signals. In fact, after a few iterations, the ISI almost totally eliminated and the performance is close to the matched filter bound (MFB). Moreover, it can be observed that when the channels between the BS antennas and the user are uncorrelated, the performance is worse than when they are equal. This can be explained by the fact that although in average the high-power signal is associated with a better channel, the situation can be inverted locally along the signal bandwidth due the existence of deep fades. This effect is illustrated in Fig. 3, which shows the channel frequency responses associated to a given user when the PSR is $\beta=14 \mathrm{~dB}$. More concretely, Fig. 3(a) shows the evolution of the channel frequency responses when there are different and uncorrelated channels between the BS antennas and the user and Fig. 3(b) shows equal channels. ${ }^{3}$ As can be observed in the figure, when the channels are different and uncorrelated, the "best channel" can be worse than the channel associated to the low-power signal in some subcarriers. Indeed, although this is a rare event, it can lead to performance degradation.

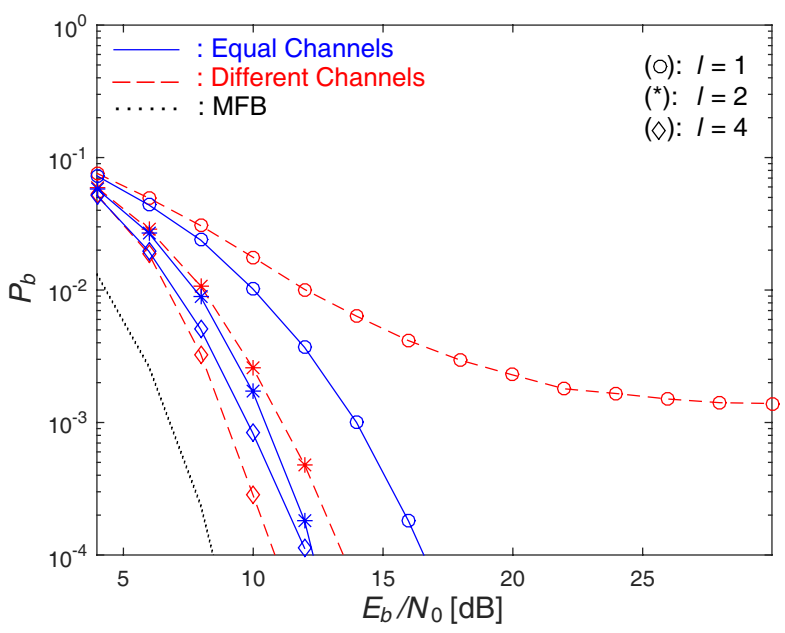

Figure 2: BER performance for the furthest user.

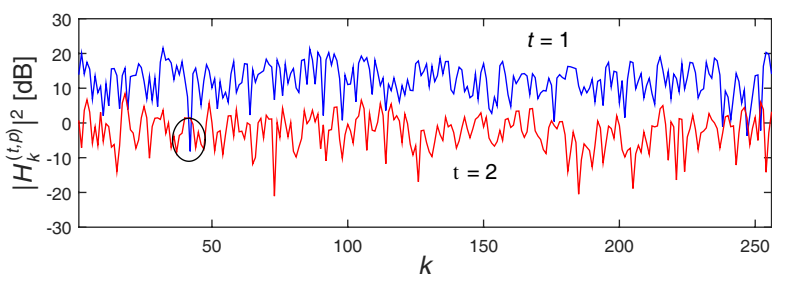

(a)

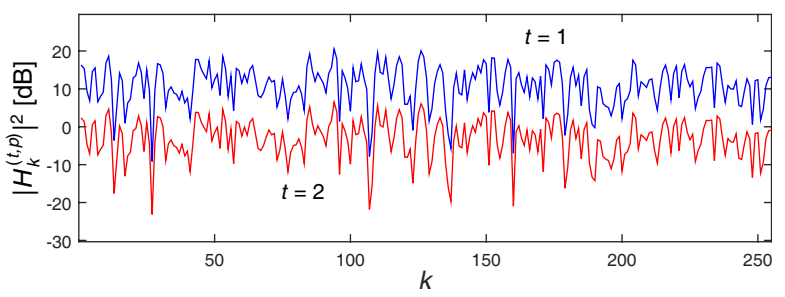

(b)

Figure 3: Evolution of the channel frequency response at a given user considering: (a) different channels and (b) equal channels.

Figure 4 shows the BER associated with the closest user, considering uncorrelated channels, IBDFE receivers with $L=4$ iterations and a PSR of $\beta=14 \mathrm{~dB}$. From the figure, it can be noted that even when the channels between the BS antennas and the closest user are uncorrelated, the users can be efficiently separated and the performance associated with the closest user can approximate the MFB. In fact, although when only one iteration (i.e., $l=1$ ) is considered and we have a linear FDE receiver the performance deviates considerably from the MFB, when $l=4$ the BER improves substantially.

Figure 5 shows the BER for the closest user, considering equal channels, IB-DFE receivers with $L=4$ iterations and a PSR of $\beta=14 \mathrm{~dB}$. As expected, when the channels between the BS transmit antennas and the closest user are equal, the performance associated with the data detection can approximate the MFB.

To understand the choice of a power separation of $\beta=14 \mathrm{~dB}$, it is important to compare the performance of our technique to the one associated with the ideal case, i.e., when the closest user knows the data transmitted to the furthest user and performs an ideal interference cancellation. Fig. 6 depicts the BER performance of the closest user considering $\beta=14 \mathrm{~dB}$ as well as the ideal scenario, where there is perfect cancellation of the IUI from the interfering signal (i.e., the high power signal). Clearly, the use of $\beta=14 \mathrm{~dB}$ leads to a BER performance that is almost equal to the one obtained in the ideal scenario, i.e., when the closest user makes a perfect IUI cancellation of the interfering signal from the furthest user.

Although is expected that the use of larger values of $\beta$ helps the user separation and leads to a better IUI cancellation, it is important to study what is the impact of $\beta$ on the performance. Fig. 7 ,

\footnotetext{
${ }^{3}$ Actually, the channels have a little difference that is constant along the bandwidth of the signals.
} 


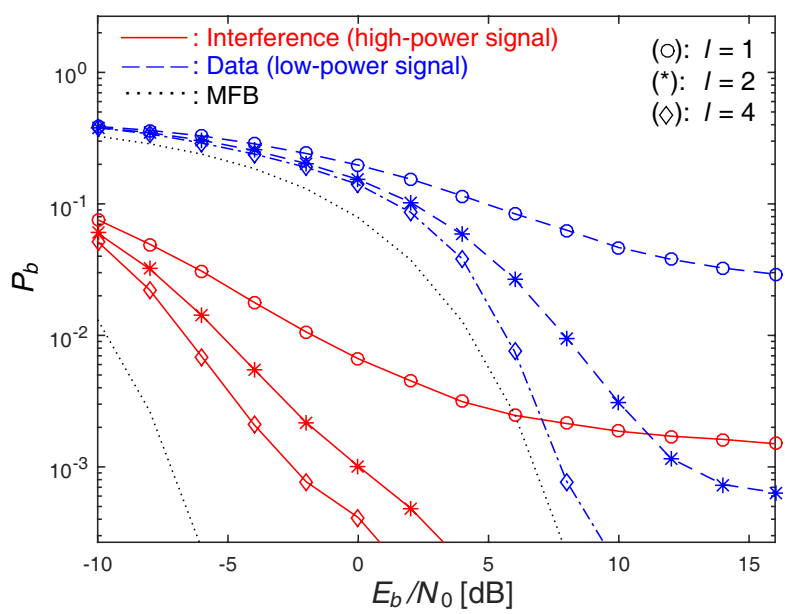

Figure 4: BER performance for the closest user considering different channels.

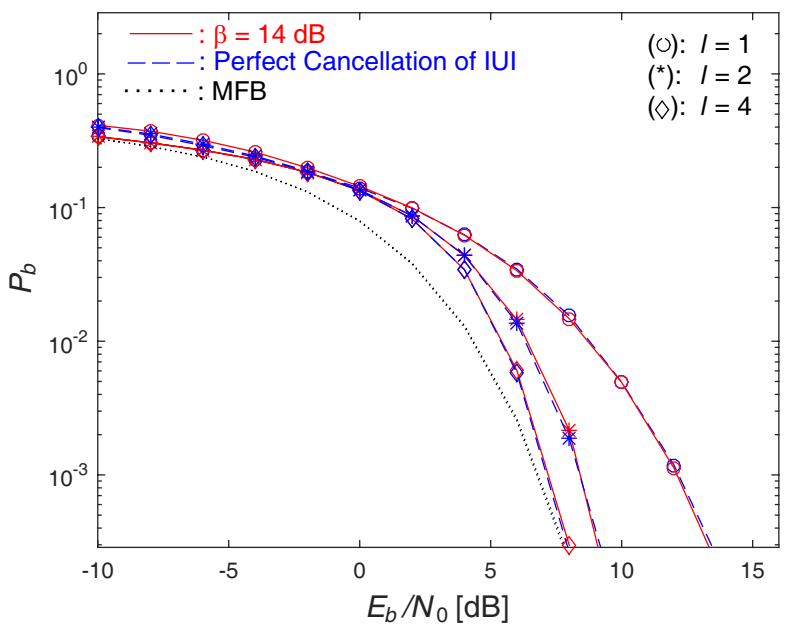

Figure 6: BER performance associated to the closest user considering $\beta=6 \mathrm{~dB}$ and perfect cancellation of the interfering signal

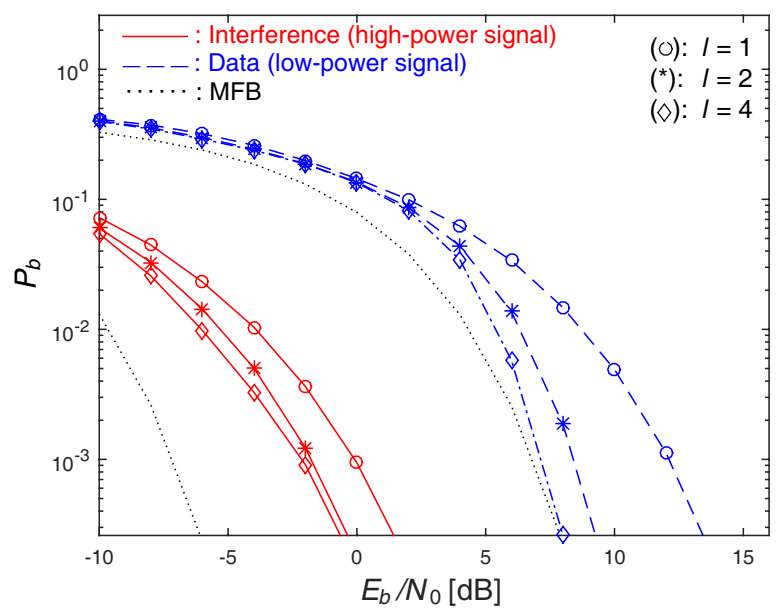

Figure 5: BER performance for the closest user considering equal channels.

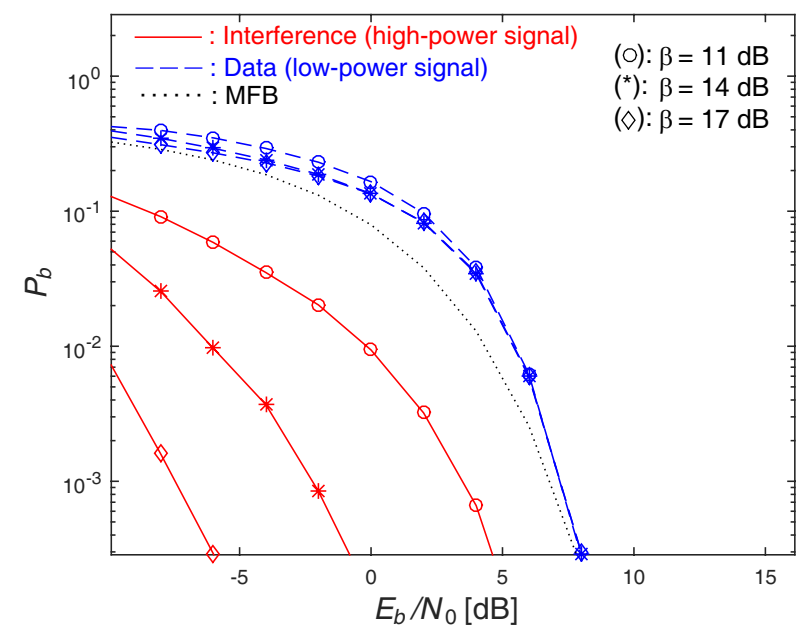

Figure 7: BER performance associated to the closest user considering different values of $\beta$.

which shows the impact of the PSR $\beta$ on the BER of the closest user considering equal channels (it is assumed that $L=4$ but only the fourth IB-DFE iteration $(l=4)$ is shown). As stated before, a PSR of $\beta=14 \mathrm{~dB}$ is high enough to separate the interfering signal of the furthest user from the data signal of the closest user and the BER associated with the closest user is close to the MFB. In fact, this justify our choice for the simulations presented above (see Figs. 4 and 5) although, however, one can also see that the performance gains relatively to the case where $\beta=11 \mathrm{~dB}$ are low and only observable at high BER values. This means that considering higher values of $\beta$ does not significantly increase the performance (as can be observed when $\beta=17 \mathrm{~dB}$ ) and only reduces the energy efficiency of the NOMA system.

\section{CONCLUSIONS}

In this paper we considered the use of SC-FDE signals combined with NOMA schemes in the downlink transmission. We presented a multi-antenna transmission technique that is compatible with the use of high-efficiency, nonlinear power amplifiers and does not require any pre-processing and allows the use of power-efficient amplifiers. Moreover, an efficient detection is achieved using iterative receivers with joint multi-user detection and equalization. Our performance results showed that the prosed technique allows high power efficiency, with BER performance close to the MFB. 


\section{ACKNOWLEDGMENT}

This work was supported in part by Fundação para a Ciência e Tecnologia and Instituto de Telecomunicaçòes under projects UID/EEA/50008/2019 and PES3N POCI-01-0145-FEDER-030629.

\section{REFERENCES}

1. Saito, Y., et al., "Non-orthogonal multiple access (NOMA) for future radio access," IEEE Vehicular Technology Conference (VTC) Spring'13, 1-5, Sept. 2013.

2. Shin, W., et al., "Non-orthogonal multiple access in multi-cell networks: Theory, performance, and practical challenges," CoRR, Vol. abs/1611.01607, 2016.

3. Saito, Y., et al., "System level performance evaluation of downlink non-orthogonal multiple access (NOMA)," IEEE Symposium on Personal, Indoor and Mobile Radio Communications (PIMRC), Sept. 2013.

4. Dai, L., B. Wang, and Y. Yuan, "Non-orthogonal multiple access for 5G: Solutions, challenges, opportunities, and future research trends," IEEE Commun. Mag., Vol. 53, No. 9, 74-81, Sept. 2015.

5. Ding, Z., Z. Yang, P. Fan, and H. V. Poor, "On the performance of non-orthogonal multiple access in 5G systems with randomly deployed users," IEEE Signal Process. Lett., Vol. 21, No. 12, 1501-1505, Dec. 2014.

6. Ding, Z., Y. Liu, J. Choi, Q. Sun, M. Elkashlan, C.-L. I, and H. V. Poor, "Application of nonorthogonal multiple access in LTE and 5G networks," IEEE Commun. Mag., Vol. 55, No. 2, 185-191, Feb. 2017.

7. Ding, Z., L. Dai, R. Schober, and H. Poor, "NOMA meets finite resolution analog beamforming in massive MIMO and millimeter-wave networks," IEEE Commun. Lett., Vol. PP, May 2017.

8. Islam, S., N. Avazov, O. Dobre, and K. Kwak, "Power-domain non-orthogonal multiple access (NOMA) in 5G systems: Potentials and challenges," IEEE Commun. Surveys and Tuts., Vol. 19, No. 2, 721-742, 2017.

9. Zhang, J., X. Wang, T. Hasegawa, and T. Kubo, "Downlink non-orthogonal multiple access (NOMA) constellation rotation," IEEE VTC'2016 (Fall), Sept. 2016.

10. Gomes, M., R. Dinis, V. Silva, F. Cercas, and M. Tomlinson, "Error rate analysis of M-PSK with magnitude modulation envelope control," IET Electronic Lett., Vol. 49, No. 18, 11841186, Aug. 2013.

11. Luzio, M., R. Dinis, and P. Montezuma, "Pragmatic frequency domain equalization for single carrier with offset modulations," IEEE Trans. Wireless Commun., Vol. 9, No. 12, 4496-4505, Dec. 2013.

12. Raab, H., et al., "Power amplifiers and transmitters for RF and microwave," IEEE Trans. Microw. Theory Techn., Vol. 50, No. 3, 814-826, Mar. 2005.

13. Benvenuto, N. and S. Tomasin, "Block iterative DFE for single carrier modulation," Electronics Letters, Vol. 38, No. 19, 1144-1145, Sept. 2002.

14. Benvenuto, N., R. Dinis, D. Falconer, and S. Tomasin, "Single carrier modulation with nonlinear frequency domain equalization: An idea whose time has come again," Proc. IEEE, Vol. 98, No. 1, 69-96, Jan. 2010. 